TRANSACTIONS OF THE

AMERICAN MATHEMATICAL SOCIETY

Volume 352 , Number 5 , Pages $2283-2299$

S 0002-9947(00)02542-3

Article electronically published on February 16, 2000

\title{
FROM THE RESTRICTED TO THE FULL THREE-BODY PROBLEM
}

\author{
KENNETH R. MEYER AND DIETER S. SCHMIDT
}

To Hugh Turrittin on his ninety birthday

\begin{abstract}
The three-body problem with all the classical integrals fixed and all the symmetries removed is called the reduced three-body problem. We use the methods of symplectic scaling and reduction to show that the reduced planar or spatial three-body problem with one small mass is to the first approximation the product of the restricted three-body problem and a harmonic oscillator. This allows us to prove that many of the known results for the restricted problem have generalizations for the reduced three-body problem.

For example, all the non-degenerate periodic solutions, generic bifurcations, Hamiltonian-Hopf bifurcations, bridges and natural centers known to exist in the restricted problem can be continued into the reduced three-body problem. The classic normalization calculations of Deprit and Deprit-Bartholomé show that there are two-dimensional KAM invariant tori near the Lagrange point in the restricted problem. With the above result this proves that there are three-dimensional KAM invariant tori near the Lagrange point in the reduced three-body problem.
\end{abstract}

\section{INTRODUCTION}

There is a vast literature on the restricted three-body problem. Among other things, there are investigations of the equilibriums points and their stability, investigations of the existence, stability and bifurcation of periodic orbits, and investigations of collisions and ejection orbits. The restricted problem is said to be a limit of the three-body problem as one of the masses tends to zero, and so to each result for the restricted problem there should be a corresponding result for the full three-body problem. Indeed, there are many such results for the three-body problem, but usually the proofs for the three-body problem are similar to, but independent of, the proofs for the restricted problem. The goal of this paper is to set up a framework whereby results established in the restricted problem carry over to the three-body problem with little or no effort. Some of the results given below are new and some are not, but we think the method of proof is different.

By the restricted problem we shall mean the circular, planar or spatial, restricted three-body problem, and by the full problem we shall mean the planar or spatial

Received by the editors July 22, 1997 and, in revised form, January 20, 1998.

2000 Mathematics Subject Classification. Primary 70F05, 37N05.

Key words and phrases. Restricted three-body problem, three-body problem, reduction, symplectic scaling, normal forms, KAM theory.

This research was partially supported by grants from the National Science Foundation and the Taft Foundation. 
three-body problem. The restricted problem is a Hamiltonian system of differential equations which describes the motion of an infinitesimal particle (the satellite) moving under the gravitational influence of two particles of finite mass (the primaries) which are moving on a circular orbit of the Kepler problem.

Since the motion of the primaries is given, the restricted problem has two degrees of freedom for the planar problem and three degrees of freedom for the spatial problem. However, the full problem has six degrees of freedom in the planar case and nine degrees of freedom in the spatial case. Thus, at first the restricted problem seems too small to reflect the full complexity of the full problem; but when the symmetries of the full problem are taken into account the dimension gap narrows considerably.

The Hamiltonian of the full problem is invariant under Euclidean motions, i.e. translations and rotations, which begets the integrals of linear and angular momentum. Translations and rotations give rise to ignorable coordinates. Holding the integrals fixed and dropping the ignorable coordinates reduces the full problem from six to three degrees of freedom in the planar case and from nine to four degrees of freedom in the spatial case. Thus the full problem on the reduced space is only one degree of freedom larger than the restricted problem in either the planar or the spatial case. We shall call the full three-body problem on the reduced space the reduced three-body problem or simply the reduced problem.

The question addressed in this paper is the relation between the reduced problem with one small mass and the restricted three-body problem. The goal is to illustrate a set of methods and results that assure that much of what is known about the restricted problem can be carried over to the reduced problem. We will not attempt to delineate every such result, since this would be too tedious. Once a few examples have been seen the general pattern can be gleaned.

In Section 2 we use the methods of symplectic scaling and reduction to show that the reduced planar or spatial three-body problem with one small mass is to the first approximation the product of the restricted three-body problem and a harmonic oscillator. From this it follows easily in Section 3 that a nondegenerate periodic solution of the restricted problem can be continued into the reduced problem. We show in Section 6 that a periodic solution of general elliptic type (with a twist) can be continued into the reduced three-body problem, and so there are three-dimensional invariant KAM tori in the reduced three-body problem near the Lagrange triangular point. We illustrate in Sections 4 and 5 how many bifurcation results can be continued into the reduced problem. For example, the HamiltonianHopf bifurcation occurs in the reduced three-body problem.

\section{SCALING AND REDUCTION}

In this section we make a series of symplectic changes of variables in the threebody problem which show how to look at the restricted problem as the limit of the reduced problem with one small mass. To the first approximation the reduced problem with one small mass is separable, i.e. the Hamiltonian of the reduced problem to the first approximation is the sum of the Hamiltonian of the restricted problem and the Hamiltonian of a harmonic oscillator. This result was established in [18, 20] for the planar problem, so we shall consider only the spatial case here.

The three-body problem in three-dimensional space has nine degrees of freedom. By placing the center of mass at the origin and setting linear momentum equal to 
zero the problem reduces one with six degrees of freedom. This is easily done using Jacobi coordinates. The Hamiltonian of the three-body problem in rotating (about the $z$-axis) Jacobi coordinates $\left(u_{0}, u_{1}, u_{2}, v_{0}, v_{1}, v_{2}\right)$ is

$$
\begin{aligned}
H= & \frac{\left\|v_{0}\right\|^{2}}{2 M_{0}}-u_{0}^{T} J v_{0}+\frac{\left\|v_{1}\right\|^{2}}{2 M_{1}}-u_{1}^{T} J v_{1}-\frac{m_{0} m_{1}}{\left\|u_{1}\right\|} \\
& +\frac{\left\|v_{2}\right\|^{2}}{2 M_{2}}-u_{2}^{T} J v_{2}-\frac{m_{1} m_{2}}{\left\|u_{2}-\alpha_{0} u_{1}\right\|}-\frac{m_{2} m_{0}}{\left\|u_{2}+\alpha_{1} u_{1}\right\|}
\end{aligned}
$$

where $u_{i}, v_{i} \in \mathbb{R}^{3}$,

$$
\begin{gathered}
M_{0}=m_{0}+m_{1}+m_{2}, \quad M_{1}=m_{0} m_{1} /\left(m_{0}+m_{1}\right), \\
M_{2}=m_{2}\left(m_{0}+m_{1}\right) /\left(m_{0}+m_{1}+m_{2}\right), \\
\alpha_{0}=m_{0} /\left(m_{0}+m_{1}\right), \quad \alpha_{1}=m_{1} /\left(m_{0}+m_{1}\right),
\end{gathered}
$$

and

$$
J=\left(\begin{array}{ccc}
0 & 1 & 0 \\
-1 & 0 & 0 \\
0 & 0 & 0
\end{array}\right)
$$

In these coordinates $u_{0}$ is the center of mass, $v_{0}$ is total linear momentum, and total angular momentum is

$$
A=u_{0} \times v_{0}+u_{1} \times v_{1}+u_{2} \times v_{2} .
$$

See Meyer and Hall [20] for details.

The set where $u_{0}=v_{0}=0$ is invariant, and setting these two coordinates to zero effects the first reduction. Setting $u_{0}=v_{0}=0$ reduces the problem by three degrees of freedom.

We consider angular momentum to be nonzero. One way to reduce the problem by two more degrees is to hold the vector $A$ fixed and eliminate the rotational symmetry about the $A$ axis. Another way to reduce the problem is to note that $A_{z}$, the $z$-component of angular momentum, and $\mathbf{A}=\|A\|$, the magnitude of angular momentum, are integrals in involution. Two independent integrals in involution can be used to reduce a system by two degrees of freedom, see [34]. In either case we pass to the reduced space as defined in Meyer [19] or Marsden and Weinstein [13].

Assume that one of the particles has small mass by setting $m_{2}=\varepsilon^{2}$, where $\varepsilon$ is to be considered as a small parameter. Also set $m_{0}=\mu, m_{1}=1-\mu$ and $\nu=\mu(1-\mu)$, so that

$$
\begin{gathered}
M_{1}=\nu=\mu(1-\mu), \quad M_{2}=\varepsilon^{2} /\left(1+\varepsilon^{2}\right)=\varepsilon^{2}-\varepsilon^{4}+\cdots . \\
\alpha_{0}=\mu, \quad \alpha_{1}=1-\mu .
\end{gathered}
$$

The Hamiltonian becomes

$$
H=K+\tilde{H},
$$

where

$$
K=\frac{1}{2 \nu}\left\|v_{1}\right\|^{2}-u_{1}^{T} J v_{1}-\frac{\nu}{\left\|u_{1}\right\|}
$$


and

$$
\tilde{H}=\frac{\left(1+\varepsilon^{2}\right)}{2 \varepsilon^{2}}\left\|v_{2}\right\|^{2}-u_{2}^{T} J v_{2}-\frac{\varepsilon^{2}(1-\mu)}{\left\|u_{2}-\mu u_{1}\right\|}-\frac{\varepsilon^{2} \mu}{\left\|u_{2}+(1-\mu) u_{1}\right\|} .
$$

$K$ is the Hamiltonian of the Kepler problem in rotating coordinates. We can simplify $K$ by making the scaling $u_{i} \rightarrow u_{i}, v_{i} \rightarrow \nu v_{i}, K \rightarrow \nu^{-1} K, \tilde{H} \rightarrow \nu^{-1} \tilde{H}$, $\varepsilon^{2} \nu^{-1} \rightarrow \varepsilon^{2}$, so that

$$
K=\frac{1}{2}\left\|v_{1}\right\|^{2}-u_{1}^{T} J v_{1}-\frac{1}{\left\|u_{1}\right\|}
$$

and

$$
\tilde{H}=\frac{\left(1+\nu \varepsilon^{2}\right)}{2 \varepsilon^{2}}\left\|v_{2}\right\|^{2}-u_{2}^{T} J v_{2}-\frac{\varepsilon^{2}(1-\mu)}{\left\|u_{2}-\mu u_{1}\right\|}-\frac{\varepsilon^{2} \mu}{\left\|u_{2}+(1-\mu) u_{1}\right\|} .
$$

$K$ has a critical point at $u_{1}=a=(1,0,0)^{T}, v_{1}=b=(0,1,0)^{T}$ - it corresponds to a circular orbit of the Kepler problem. Expand $K$ in a Taylor series about this point, ignore the constant term, and make the scaling

$$
u_{1} \rightarrow a+\varepsilon q, \quad v_{1} \rightarrow b+\varepsilon p, \quad K \rightarrow \varepsilon^{-2} K
$$

to get $K=K_{0}+O(\varepsilon)$, where

$$
K_{0}=\frac{1}{2}\left(p_{1}^{2}+p_{2}^{2}+p_{3}^{2}\right)+q_{2} p_{1}-q_{1} p_{2}+\frac{1}{2}\left(-2 q_{1}^{2}+q_{2}^{2}+q_{3}^{2}\right) .
$$

Now scale $\tilde{H}$ by the above and

$$
u_{2}=\xi, \quad v_{2}=\varepsilon^{2} \eta, \quad \tilde{H} \longrightarrow \varepsilon^{-2} \tilde{H} .
$$

The totality is a symplectic scaling with multiplier $\varepsilon^{-2}$, and so the Hamiltonian of the three-body problem becomes $H_{R}+K_{0}+O(\varepsilon)$, where $K_{0}$ is given in (4) and $H_{R}$ is the Hamiltonian of the restricted problem, i.e.

$$
H_{R}=\frac{1}{2}\|\eta\|^{2}-\xi^{T} J \eta-\frac{(1-\mu)}{\|\xi-(\mu, 0,0)\|}-\frac{\mu}{\|\xi+(1-\mu, 0,0)\|} .
$$

To obtain the expansions above, recall that $u_{1}=(1,0,0)+O(\varepsilon)$.

We have already reduced the problem by using the transitional invariance and the conservation of linear momentum, so now we will complete the reduction by using the rotational invariance and the conservation of angular momentum.

Recall that angular momentum in the original coordinates is $A=u_{1} \times v_{1}+u_{2} \times v_{2}$ and in the scaled coordinates it becomes

$$
A=(a+\varepsilon q) \times(b+\varepsilon p)+\varepsilon^{2} \xi \times \eta,
$$

and so holding angular momentum fixed by setting $A=a \times b$ imposes the constraint

$$
0=a \times p+q \times b+O(\varepsilon)=\left(-q_{3},-p_{3}, p_{2}+q_{1}\right)+O(\varepsilon) .
$$

Now let us do the reduction when $\varepsilon=0$, so that the Hamiltonian is $H=H_{R}+K_{0}$ and holding angular momentum fixed is equivalent to $q_{3}=p_{3}=p_{2}+q_{1}=0$. Notice that the angular momentum constraint is only on the $q, p$ variables. Make 
the symplectic change of variables

$$
\begin{array}{ll}
r_{1}=q_{1}+p_{2}, & R_{1}=p_{1}, \\
r_{2}=q_{2}+p_{1}, & R_{2}=p_{2}, \\
r_{3}=q_{3}, & R_{3}=p_{3},
\end{array}
$$

so that

$$
K_{0}=\frac{1}{2}\left(r_{2}^{2}+R_{2}^{2}\right)+\frac{1}{2}\left(r_{3}^{2}+R_{3}^{2}\right)+r_{1} R_{2}-r_{1}^{2} .
$$

Notice that holding angular momentum fixed in these coordinates is equivalent to $r_{1}=r_{3}=R_{3}=0$, that $R_{1}$ is an ignorable coordinate, and that $r_{1}$ is an integral. Thus passing to the reduced space reduces $K_{0}$ to

$$
K_{0}=\frac{1}{2}\left(r_{2}^{2}+R_{2}^{2}\right) .
$$

Thus when $\varepsilon=0$ the Hamiltonian of the reduced three-body problem becomes

$$
H=H_{R}+\frac{1}{2}\left(r^{2}+R^{2}\right),
$$

which is the sum of the Hamiltonian of the restricted three-body problem and a harmonic oscillator. Here in (11) and henceforth we have dropped the subscript 2. The equations and integrals all depend smoothly on $\varepsilon$, and so for small $\varepsilon$ the Hamiltonian becomes

$$
H=H_{R}+\frac{1}{2}\left(r^{2}+R^{2}\right)+O(\varepsilon) .
$$

We can also introduce action-angle variables $(I, \iota)$ by

$$
r=\sqrt{2 I} \cos \iota, \quad R=\sqrt{2 I} \sin \iota,
$$

to give

$$
H=H_{R}+I+O(\varepsilon) .
$$

The reduced three-body problem in two or three dimensions with one small mass is approximately the product of the restricted problem and a harmonic oscillator.

\section{Continuation of PeRiodic SOlutions}

A periodic solution of a conservative Hamiltonian system always has the characteristic multiplier +1 with algebraic multiplicity at least 2 . If the periodic solution has the characteristic multiplier +1 with algebraic multiplicity exactly equal to 2 , then the periodic solution is called non-degenerate or sometimes elementary. A non-degenerate periodic solution lies in a smooth cylinder of periodic solutions which are parameterized by the Hamiltonian. Moreover, if the Hamiltonian depends smoothly on parameters, then the periodic solution persists for small variations of the parameters. See Meyer and Hall [20, pages 133-36 and 155-56, for complete details.

Theorem. A nondegenerate periodic solution of the planar or spatial restricted three-body problem whose period is not a multiple of $2 \pi$ can be continued into the reduced three-body problem.

More precisely: 
Theorem. Let $\eta=\phi(t), \xi=\psi(t)$ be a periodic solution with period $T$ of the restricted problem whose Hamiltonian is (5). Let its multipliers be $+1,+1, \beta, \beta^{-1}$ in the planar case or $+1,+1, \beta_{1}, \beta_{1}^{-1}, \beta_{2}, \beta_{2}^{-1}$ in the spatial case. Assume that $T \neq n 2 \pi$ for all $n \in \mathbb{Z}$, and $\beta \neq+1$ in the planar case or $\beta_{1} \neq+1$ and $\beta_{2} \neq+1$ in the spatial case. Then the reduced three-body problem, the system with Hamiltonian (12), has a periodic solution of the form $\eta=\phi(t)+O(\varepsilon), \xi=\psi(t)+O(\varepsilon), r=O(\varepsilon), R=O(\varepsilon)$ whose period is $T+O(\varepsilon)$. Moreover, its multipliers are $+1,+1, \beta+O(\varepsilon), \beta^{-1}+$ $O(\varepsilon), e^{i T}+O(\varepsilon), e^{-i T}+O(\varepsilon)$ in the planar case, or $+1,+1, \beta_{1}+O(\varepsilon), \beta_{1}^{-1}+O(\varepsilon), \beta_{2}+$ $O(\varepsilon), \beta_{2}^{-1}+O(\varepsilon), e^{i T}+O(\varepsilon), e^{-i T}+O(\varepsilon)$ in the spatial case.

Proof. When $\varepsilon=0$ the reduced problem with Hamiltonian (12) has the periodic solution $\eta=\phi(t), \xi=\psi(t), r=0, R=0$ with period $T$. Its multipliers are $+1,+1, \beta, \beta^{-1}, e^{i T}, e^{-i T}$ in the planar case or $+1,+1, \beta_{1}, \beta_{1}^{-1}, \beta_{2}, \beta_{2}^{-1}, e^{i T}, e^{-i T}$ in the spatial case. By the assumption $T \neq n 2 \pi$ it follows that $e^{ \pm i T} \neq+1$, and so this periodic solution is non-degenerate. The classical continuation theorem can be applied to show that this solution can be continued smoothly into the problem with $\varepsilon$ small and non-zero. See [5], page 142, or [20], pages 154-56.

The planar version of this theorem is due to Hadjidemetriou [11. There are similar theorems about non-degenerate symmetric periodic solutions - see [18.

There are three classes of non-degenerate periodic solutions of the planar restricted problem that are obtained by continuation of the circular orbits of the Kepler problem using a small parameter. The small parameter might be $\mu$, the mass ratio parameter, giving the periodic solutions of the first kind of Poincaré [32, 27], a small distance giving Hill's lunar orbits [4, 7, 32], or a large distance giving the comet orbits [17, 24]. All these papers cited except [17] use a symmetry argument, and so do not calculate the multipliers. However, in Meyer and Hall [20] a unified treatment of all three cases is given, and the multipliers are computed and found to be nondegenerate. Thus, there are three corresponding families of periodic solutions of the reduced problem. The corresponding results with independent proofs for the reduced problem are found in [14, 18, 23, 24, 26, 31].

One of the most interesting families of nondegenerate periodic solution of the spatial restricted problem can be found in Belbruno [3]. He regularized double collisions when $\mu=0$, and showed that some spatial collision orbits are nondegenerate periodic solutions in the regularized coordinates. Thus, they can be continued into the spatial restricted problem as nondegenerate periodic solutions for $\mu \neq 0$. Now these same orbits can be continued into the reduced three-body problem.

\section{Continuation of Bifurcating Periodic solutions}

Many families of periodic solutions of the restricted problem have been studied, and numerous bifurcations have been observed. Most of these bifurcations are 'generic one parameter bifurcations' as defined in [15] (see also [20, Chapter VIII). Other bifurcations seem to be generic in either the class of symmetric solutions or generic two-parameter bifurcations. We claim that these bifurcations can be carried over to the reduced three-body problem, mutatis mutandis. Since there are a multitude of different bifurcations and they are all generalize in a similar manner, we shall illustrate only one simple case - the 3-bifurcation of [15], called the phantom kiss in [1]. 
Let $p(t, h)$ be a smooth family of non-degenerate periodic solutions of the restricted problem parameterized by $H_{R}$, i.e. $H_{R}(p(t, h))=h$, with period $\tau(h)$. When $h=h_{0}$ let the periodic solution be $p_{0}(t)$ with period $\tau_{0}$, so $p_{0}(t)=p\left(t, h_{0}\right)$ and $\tau_{0}=\tau\left(h_{0}\right)$. We will say that the $\tau_{0}$-periodic solution $p_{0}(t)$ of the restricted problem is a 3 -bifurcation orbit if the cross section map $(\psi, \Psi) \longrightarrow\left(\psi^{\prime}, \Psi^{\prime}\right)$ in the surface $H_{R}=h$ for this periodic orbit can be put into the normal form

$$
\begin{aligned}
& \psi^{\prime}=\psi+(2 \pi k / 3)+\alpha\left(h-h_{0}\right)+\beta \Psi^{1 / 2} \cos (3 \psi)+\cdots, \\
& \Psi^{\prime}=\Psi-2 \beta \Psi^{3 / 2} \sin (3 \psi)+\cdots, \\
& T=\tau_{0}+\cdots,
\end{aligned}
$$

and $k=1,2$, and $\alpha$ and $\beta$ are non-zero constants. In the above $\psi, \Psi$ are normalized action-angle coordinates in the cross section intersect $H_{R}=h$, and $T$ is the first return time for the cross section. The periodic solution, $p(t, h)$, corresponds to the point $\Psi=0$. The multipliers of the periodic solution $p_{0}(t)$ are $+1,+1, e^{+2 k \pi i / 3}, e^{-2 k \pi i / 3}$ (cube roots of unity) so the periodic solution is a nondegenerate elliptic periodic solution. Thus, this family of periodic solutions can be continued into the reduced problem, provided $\tau_{0}$ is not a multiple of $2 \pi$, by the result of the last subsection.

The above assumptions imply that the periodic solution $p(t, h)$ of the restricted problem undergoes a bifurcation. In particular, there is a one parameter family $p_{3}(t, h)$ of hyperbolic periodic solution of period $3 \tau_{0}+\cdots$ whose limit is $p_{0}(t)$ as $h \longrightarrow h_{0}$. See [16, 20] for complete details.

Theorem. Let $p_{0}(t)$ be a 3-bifurcation orbit of the restricted problem that is not in resonance with the harmonic oscillator, i.e. assume that $3 \tau_{0} \neq 2 n \pi$, for $n \in \mathbb{Z}$. Let $\tilde{p}(t, h, \varepsilon)$ be the $\tilde{\tau}(h, \varepsilon)$-periodic solution which is the continuation into the reduced problem of the periodic solution $p(t, h)$ for small $\varepsilon$. Thus $\tilde{p}(t, h, \varepsilon) \longrightarrow(p(t, h), 0,0)$ and $\tilde{\tau}(h, \varepsilon) \longrightarrow \tau(h)$ as $\varepsilon \longrightarrow 0$.

Then there is a smooth function $\tilde{h}_{0}(\varepsilon)$ with $\tilde{h}_{0}(0)=h_{0}$ such that $\tilde{p}\left(t, \tilde{h}_{0}(\varepsilon), \varepsilon\right)$ has multipliers $+1,+1, e^{+2 k \pi i / 3}, e^{-2 k \pi i / 3}, e^{+\tau i}+O(\varepsilon), e^{-\tau i}+O(\varepsilon)$, i.e. exactly one pair of multipliers are cube roots of unity. Moreover, there is a family of periodic solutions of the reduced problem, $\tilde{p}_{3}(t, h, \varepsilon)$ with period $3 \tilde{\tau}(h, \varepsilon)+\cdots$ such that $\tilde{p}_{3}(t, h, \varepsilon) \longrightarrow\left(p_{3}(t, h), 0,0\right)$ as $\varepsilon \longrightarrow 0$ and $\tilde{p}_{3}(t, h, \varepsilon) \longrightarrow \tilde{p}\left(t, \tilde{h}_{0}(\varepsilon), \varepsilon\right)$ as $h \longrightarrow$ $\tilde{h}_{0}(\varepsilon)$. The periodic solutions of the family $\tilde{p}_{3}(t, h, \varepsilon)$ are hyperbolic-elliptic, i.e. they have two multipliers equal to +1 , two multipliers which are of unit modulus, and two multipliers which are real and not equal to \pm 1 .

Proof. Since the Hamiltonian of the reduced problem is $H=H_{R}+\frac{1}{2}\left(r^{2}+R^{2}\right)+O(\varepsilon)$, we can compute the cross section map for this periodic solution in the reduced problem for $\varepsilon=0$. Use $\psi, \Psi, r, R$ as coordinates in this cross section, and let $\eta=h-h_{0}$. The period map is $(\psi, \Psi, r, R) \longrightarrow\left(\psi^{\prime}, \Psi^{\prime}, r^{\prime}, R^{\prime}\right)$, where

$$
\begin{aligned}
& \psi^{\prime}=\psi^{\prime}(\psi, \Psi, r, R, \eta, \varepsilon)=\psi+(2 \pi k / 3)+\alpha \eta+\beta \Psi^{1 / 2} \cos (3 \psi)+\cdots \\
& \Psi^{\prime}=\Psi^{\prime}(\psi, \Psi, r, R, \eta, \varepsilon)=\Psi-2 \beta \Psi^{3 / 2} \sin (3 \psi)+\cdots \\
& \left(\begin{array}{c}
r^{\prime} \\
R^{\prime}
\end{array}\right)=\left(\begin{array}{c}
r^{\prime}(\psi, \Psi, r, R, \eta, \varepsilon) \\
R^{\prime}(\psi, \Psi, r, R, \eta, \varepsilon)
\end{array}\right)=B\left(\begin{array}{c}
r \\
R
\end{array}\right)+\cdots
\end{aligned}
$$


where

$$
B=\left(\begin{array}{cc}
\cos \tau & \sin \tau \\
-\sin \tau & \cos \tau
\end{array}\right)
$$

Since the periodic solution of the restricted problem is non-degenerate, it can be continued into the reduced problem, and so we may transfer the fixed point to the origin, i.e. $\Psi=r=R=0$ is fixed.

Since $\alpha \neq 0$, we can solve $\psi^{\prime}(0,0,0,0, \eta, \varepsilon)=2 \pi k / 3$ for $\eta$ as a function of $\varepsilon$ to get $\tilde{\eta}(\varepsilon)=h-\tilde{h}_{0}(\varepsilon)$. This defines the function $\tilde{h}_{0}$.

Compute the third iterate of the period map to be

$$
(\psi, \Psi, r, R) \longrightarrow\left(\psi^{3}, \Phi^{3}, r^{3}, R^{3}\right),
$$

where

$$
\begin{aligned}
& \psi^{3}=\psi+2 \pi k+3 \alpha \eta+3 \beta \Psi^{1 / 2} \cos (3 \psi)+\cdots, \\
& \Psi^{3}=\Psi-2 \beta \Psi^{3 / 2} \sin (3 \psi)+\cdots \\
& \left(\begin{array}{c}
r^{3} \\
R^{3}
\end{array}\right)=B^{3}\left(\begin{array}{c}
r \\
R
\end{array}\right)+\cdots .
\end{aligned}
$$

Since $3 \tau \neq 2 k \pi$, the matrix $B^{3}-E$ is nonsingular, where $E$ is the $2 \times 2$ identity matrix. Thus we can solve the equations $r^{3}-r=0, R^{3}-R=0$ and substitute the solutions into the equations for $\psi^{3}-\psi=0, \Psi^{3}-\Psi=0$.

The origin is always a fixed point; so, $\Psi$ is a common factor in the formula for $\Psi^{3}$. Since $\beta \neq 0$, the equation $\left(\Psi^{3}-\Psi\right) /\left(-2 \beta \Psi^{3 / 2}\right)=\sin (3 \psi)+\cdots$ can be solved for six functions $\psi_{j}(\Psi, h)=j \pi / 3+\cdots, j=0,1, \ldots, 5$. For even $j, \cos 3 \psi_{j}=+1+\cdots$, and for odd $j, \cos 3 \psi_{j}=-1+\cdots$. Substituting these solutions into the $\psi$ equation gives $\left(\psi^{3}-\psi-2 k \pi\right) / 3=\alpha \eta \pm \beta \Psi^{1 / 2}+\cdots$. The equations with a plus sign have a positive solution for $\Psi$ when $\alpha \beta \eta$ is negative, and the equations with a minus sign have a positive solution for $\Psi$ when $\alpha \beta \eta$ is positive. The solutions are of the form $\Psi_{j}^{1 / 2}=\mp \alpha \eta / \beta$. Compute the Jacobian along these solutions to be

$$
\frac{\partial\left(\Psi^{3}, \psi^{3}\right)}{\partial(\Psi, \psi)}=\left(\begin{array}{cc}
1 & 0 \\
0 & 1
\end{array}\right)+\left(\begin{array}{cc}
0 & \mp 6 \beta \Psi_{j}^{3 / 2} \\
\pm(3 \beta / 2) \Psi_{j}^{-1 / 2} & 0
\end{array}\right),
$$

and so the multipliers are $1 \pm 3 \alpha^{2} \eta^{2}$, and the periodic points are all hyperbolicelliptic.

There are many other types of generic bifurcations, e.g. Hamiltonian saddlenode bifurcation, period doubling, $k$-bifurcations with $k>3$, etc., as listed in 15, 20. If such a bifurcation occurs in the restricted problem and the period of the basic periodic orbit is not a multiple of $2 \pi$, then a similar bifurcation takes place in the reduced problem also. The proofs will be essentially the same as the proof given above.

\section{The Bifurcations at $\mathcal{L}_{4}$}

The restricted problem has five equilibrium points, of which three lie on the line joining the masses (the Eulerian equilibria) and two at the vertices of the two equilateral triangles whose base is the segment joining the primaries (the Lagrange equilibria) - see [20]. These later two equilibria are usually denoted by $\mathcal{L}_{4}, \mathcal{L}_{5}$. 
By the symmetry of the problem it is enough to consider just $\mathcal{L}_{4}$. For $0<\mu \leq$ $\mu_{1}=27 \mu(1-\mu) / 4$ the linearized equations at $\mathcal{L}_{4}$ have pure imaginary eigenvalues $\pm i \omega_{1}, \pm i \omega_{2}$. These pure imaginary eigenvalues give rise to two families of periodic solutions emanating fron the equilibria for most values of $\mu, 0<\mu<\mu_{1}$. These families are known as the Lyapunov families.

As an application of the previous result, consider the Lyapunov families of periodic solutions emanating from $\mathcal{L}_{4}$ in the restricted problem. Except as noted below, these two families have continuations into the reduced problem as Lyapunov families emanating from $\mathcal{L}_{4}$.

There are a multitude of interesting bifurcations that occur at the Lagrange equilateral triangular equilibria, $\mathcal{L}_{4}$, in the restricted problem as the mass ratio parameter $\mu$ is varied. We shall look at just a few of these interesting bifurcations to illustrate how many of the results that have been established in the restricted three-body problem can be carried over to the reduced three-body problem. Here we shall consider only the planar problem, since there is a wealth of literature on the planar restricted problem. Again we hope that the reader will realize that similar results too numerous to expound will follow in a similar manner for both the planar and spatial problems.

5.1. Linear analysis at $\mathcal{L}_{4}$. Recall [20] that the characteristic polynomial of the linearized equations at the equilibrium point $\mathcal{L}_{4}$ in the restricted problem is

$$
p_{R}(\lambda, \mu)=\lambda^{4}+\lambda^{2}+\frac{27}{4} \mu(1-\mu) .
$$

For $\mu>\mu_{1}$ the eigenvalues are complex, two with positive real part and two with negative real part; for $\mu<\mu_{1}$ the eigenvalues are $\pm i \omega_{1}= \pm i \omega_{1}(\mu), \pm i \omega_{2}= \pm i \omega_{2}(\mu)$, where $\omega_{1}^{2}+\omega_{2}^{2}=1, \omega_{1}^{2} \omega_{2}^{2}=27 \mu(1-\mu) / 4$ and $\omega_{1}<\omega_{2}$; for $\mu=\mu_{1}$ the eigenvalues are $\pm \sqrt{2} / 2 i, \pm \sqrt{2} / 2 i$, where $\mu_{1}=\frac{1}{2}(1-\sqrt{69} / 9)=0.0385 \ldots$ is the critical mass ratio of Routh.

Let $\mu_{r}$ denote the mass ratio at which $\omega_{1} / \omega_{2}=r$. These mass ratios are given as a function of $r$ by

$$
\mu_{r}=\frac{1}{2} \pm \frac{1}{2}\left\{1-\frac{16 r^{2}}{27\left(r^{2}+1\right)^{2}}\right\}^{1 / 2}
$$

where both signs are admissible since $0<\mu<1 . \mu_{r}$ is called a critical mass ratio of order $k$ if $r=k$ is a positive integer and a resonance mass ratio of order $(p, q)$ if $r=p / q, p>q>1$. The frequencies are $\omega_{1}=k /\left(1+k^{2}\right)^{1 / 2}, \omega_{2}=1 /\left(1+k^{2}\right)^{1 / 2}$ at $\mu_{k}$ and $\omega_{1}=p /\left(p^{2}+q^{2}\right)^{1 / 2}, \omega_{2}=q /\left(p^{2}+q^{2}\right)^{1 / 2}$ at $\mu_{p / q}$.

The reduced problem has a relative equilibrium at the Lagrange triangular configuration - recall the Hamiltonian of the reduced problem is (12) in rotating coordinates. From (12) the characteristic polynomial of the linearized equations at the Lagrange relative equilibrium is

$$
p_{F}(\lambda, \mu, \varepsilon)=p_{R}(\lambda, \mu)\left(\lambda^{2}+1\right)+O(\varepsilon) .
$$

But $\left(\lambda^{2}+1\right)$ is always a factor of the characteristic polynomial of a relative equilibrium on the reduced space, and so $\pm i$ are always characteristic exponents of the relative equilibrium, see [14]. Thus we can write

$$
p_{F}(\lambda, \mu, \varepsilon)=c(\lambda, \mu, \varepsilon)\left(\lambda^{2}+1\right),
$$


where

$$
c(\lambda, \mu, \varepsilon)=p_{R}(\lambda, \mu)+O(\varepsilon) .
$$

These polynomials are polynomials in $\lambda^{2}$, and so $c$ and $p_{R}$ are quadratics in $\lambda^{2}$. The discriminant is

$$
\Delta(\mu, \varepsilon)=1-27 \mu(1-\mu)+O(\varepsilon) .
$$

By definition $\Delta\left(\mu_{1}, 0\right)=0$, and also $\partial \Delta\left(\mu_{1}, 0\right) / \partial \mu=54 \mu_{1}-27 \neq 0$. Thus by the implicit function theorem there is a smooth function $\tilde{\mu}_{1}(\varepsilon)=\mu_{1}+O(\varepsilon)$ such that $\Delta\left(\tilde{\mu}_{1}(\varepsilon), \varepsilon\right)=0$ for small $\varepsilon$. Along the curve $\mathcal{C}_{1}=\left\{\left(\tilde{\mu}_{1}(\varepsilon), \varepsilon\right): \varepsilon\right.$ small $\}$ in the $\mu, \varepsilon^{-}$ parameter plane the linearized equations of the reduced problem at the Lagrange relative equilibrium has one pair of pure imaginary eigenvalues with multiplicity two. That is, along this curve the eigenvalues are $\pm i, \pm \tilde{\omega}(\varepsilon) i, \pm \tilde{\omega}(\varepsilon) i$, where $\tilde{\omega}(\varepsilon)=$ $\sqrt{2} i / 2+O(\varepsilon)$. For $\mu<\tilde{\mu}_{1}(\varepsilon)$ and small $\varepsilon$ the relative equilibrium has eigenvalues of the form $\pm i, \pm \tilde{\omega}_{1}= \pm \tilde{\omega}_{1}(\mu, \varepsilon), \pm \tilde{\omega}_{2}= \pm \tilde{\omega}_{2}(\mu, \varepsilon)$.

The frequencies have ratio $r, \tilde{\omega}_{1} / \tilde{\omega}_{2}=r$, if and only if $p_{F}(\lambda, \mu, \varepsilon)$ and $p_{F}(r \lambda, \mu, \varepsilon)$ have a common root, that is, if and only if

$$
\begin{aligned}
S_{r}(\mu, \varepsilon)= & \operatorname{resultant}\left(z^{2}+z+\frac{27}{4} \mu(1-\mu)+O(\varepsilon), r^{4} z^{2}+r^{2} z+\frac{27}{4} \mu(1-\mu)+O(\varepsilon)\right) \\
= & \frac{27}{16} \mu(\mu-1)\left(r^{2}-1\right)^{2}\left(27 \mu^{2} r^{4}-27 \mu r^{4}+54 \mu^{2} r^{2}\right. \\
& \left.-54 \mu r^{2}+4 r^{2}+27 \mu^{2}-27 \mu\right)+O(\varepsilon)
\end{aligned}
$$

By definition $S_{r}\left(\mu_{r}, 0\right)=0$, and Mathematica computes

$$
\frac{\partial S\left(\mu_{r}, 0\right)}{\partial \mu}=\frac{3 \sqrt{3} r^{2}\left(r^{2}-1\right) \sqrt{27 r^{4}+38 r^{2}+27}}{4\left(r^{2}+1\right)} \neq 0,
$$

for $r \neq 1$. Again the implicit function theorem gives smooth functions $\tilde{\mu}_{r}(\varepsilon)=$ $\mu_{r}+O(\varepsilon)$ such that $S_{r}\left(\tilde{\mu}_{r}(\varepsilon), \varepsilon\right)=0$. On the curve $\mathcal{C}_{r}=\left\{\left(\tilde{\mu}_{r}(\varepsilon), \varepsilon\right): \varepsilon\right.$ small $\}$ the linearized equations of the reduced problem at the Lagrange relative equilibrium have eigenvalues $\pm i, \pm \tilde{\omega}_{1} i, \pm \tilde{\omega}_{2} i$ with $\pm \tilde{\omega}_{1} i=\tilde{\omega}_{1}\left(\tilde{\mu}_{r}(\varepsilon), \varepsilon\right), \pm \tilde{\omega}_{2} i=\tilde{\omega}_{2}\left(\tilde{\mu}_{r}(\varepsilon), \varepsilon\right)$ and $\tilde{\omega}_{1} / \tilde{\omega}_{2}=r$.

For small $\varepsilon$ the linearized equations of the reduced problem have three frequencies at the Lagrangian relative equilibrium for $0<\mu<\tilde{\mu}_{1}$; namely,

$$
1>\tilde{\omega}_{1}>\tilde{\omega}_{2}
$$

with periods

$$
\tilde{T}_{0}=2 \pi<\tilde{T}_{1}=\frac{2 \pi}{\tilde{\omega}_{1}}<\tilde{T}_{2}=\frac{2 \pi}{\tilde{\omega}_{2}} .
$$

Since $\frac{1}{2}<\tilde{\omega}_{1}<1$ for small $\varepsilon$, the period $\tilde{T}_{1}$ is never an integral multiple of $\tilde{T}_{0}=2 \pi$. But $T_{2}=k T_{0}$ when $\varepsilon=0$, and

$$
\mu_{k}^{*}=\frac{1}{2}-\frac{\sqrt{3\left(16-16 k^{2}+27 k^{4}\right)}}{18 k^{2}} .
$$

Again, we apply the implicit function theorem to find functions $\tilde{\mu}_{k}^{*}(\varepsilon)=\mu_{k}^{*}+O(\varepsilon)$ such that $\tilde{T}_{2}(\varepsilon)=k 2 \pi$ when $\mu=\tilde{\mu}_{k}^{*}$. 
5.2. Lyapunov's center theorem. For fixed small $\varepsilon$ and $\mu<\mu_{1}(\varepsilon)$ the linearized equations of the reduced problem at the Lagrange relative equilibrium have three families of periodic solutions with periods $\tilde{T}_{0}=2 \pi, \tilde{T}_{1}(\mu, \varepsilon)=2 \pi / \tilde{\omega}_{1}(\mu, \varepsilon), \tilde{T}_{2}(\mu, \varepsilon)$ $=2 \pi / \tilde{\omega}_{2}(\mu, \varepsilon)$ with $\tilde{T}_{2}>\tilde{T}_{1}>\tilde{T}_{0}$. Lyapunov's center theorem [12, 20] can be applied to yield three families of periodic solutions emanating from the Lagrange relative equilibrium in the reduced problem for $\mu<\tilde{\mu}_{1}(\varepsilon)$ for $\varepsilon$ small, except in some special cases discussed here.

First of all there is always the family with the shortest period, $\tilde{T}_{0}=2 \pi$, emanating from the relative equilibrium for $\mu<\tilde{\mu}_{1}(\varepsilon), \varepsilon$ small. But this family is always at a relative equilibrium on the reduced space 14,32 . Next, since $\tilde{\omega}_{1}(\mu, \varepsilon)$ is not a multiple of $2 \pi$ for $\mu<\tilde{\mu}_{1}(\varepsilon)$ for $\varepsilon$ small, there is always a family emanating from the relative equilibrium whose limit period at the relative equilibrium is $\tilde{T}_{1}(\mu, \varepsilon)$. This family is the continuation of the short period family in the restricted problem.

Finally, there is a family whose limit period at the relative equilibrium is $\tilde{T}_{2}(\mu, \varepsilon)$ provided $0<\mu<\tilde{\mu}_{1}(\varepsilon), \mu \neq \tilde{\mu}_{k}(\varepsilon), \mu \neq \tilde{\mu}_{k}^{*}(\varepsilon), k \notin \mathbb{Z}$, for $\varepsilon$ small. This is the continuation (where it exists) of the long period family in the restricted problem. It would be interesting to see a numerical investigation of the evolution of this last family as the parameters are varied - provided the investigation is carried out with the same care as found in the classic studies [9, 25].

5.3. Hamiltonian Hopf bifurcation at $\mathcal{L}_{4}$. One of the most interesting bifurcations occurs in the restricted problem at the equilibrium point $\mathcal{L}_{4}$ as the mass ratio parameter passes through the Routh critical mass ratio $\mu_{1}$. The linearized equations at $\mathcal{L}_{4}$ have two pairs of pure imaginary eigenvalues, $\pm \omega_{1} i, \pm \omega_{2} i$ for $0<\mu<\mu_{1}$, eigenvalues $\pm i \sqrt{2} / 2$ of multiplicity two for $\mu=\mu_{1}$, and eigenvalues $\pm \alpha \pm \beta i, \alpha \neq 0$, $\beta \neq 0$, for $\mu_{1}<\mu \leq 1 / 2$. For $\mu<\mu_{1}$ and $\mu$ near $\mu_{1}$, Lyapunov's Center Theorem establishes the existence of two families of periodic solutions emanating from the equilibrium point $\mathcal{L}_{4}$, and for $\mu_{1}<\mu \leq 1 / 2$, the stable manifold theorem asserts that there are no periodic solutions near $\mathcal{L}_{4}$. What happens to these periodic solutions as $\mu$ passes through $\mu_{1}$ ?

Buchanan [6] proved, up to a small computation, that there are still two families of periodic solutions emanating from the libration point $\mathcal{L}_{4}$ even when $\mu=\mu_{1}$. This is particularly interesting, because the linearized equations have only one family. The small computation of a coefficient of a higher order term was completed by Deprit and Henrard [9], thus showing that Buchanan's theorem did indeed apply to the restricted problem. Palmore 25] investigated the question numerically and was led to the conjecture that the two families detach as a unit from the libration point and recede as $\mu$ increases from $\mu_{1}$. Finally, Meyer and Schmidt 22 established the general theorem which has become known as the Hamiltonian Hopf Bifurcation Theorem, and then established Palmore's conjecture using the calculation of Deprit and Henrard [9. Unfortunately, a spurious factor of $\sqrt{2}$ occurred in the application of Deprit's calculation, but the result holds. Also see [20], pages 218-24. Subsequently, this theorem has been reproved by several authors by essentially the same method - see for example [10] for a complete contemporary treatment of this and related problems.

First we must set up the general theorem along the lines found in [20, 22]. We will drop the explicit $\varepsilon$ dependence until it is necessary to display it, but we will indicate those variables which will depend on $\varepsilon$ with a tilde. The normal form for a quadratic Hamiltonian (linear Hamiltonian system) with eigenvalues $\pm \tilde{\omega} i$ with 
multiplicity two, which is non-simple, and $\pm i$ with multiplicity one, is

$$
Q_{0}=\tilde{\omega}\left(\xi_{2} \eta_{1}-\xi_{1} \eta_{2}\right)+(\delta / 2)\left(\xi_{1}^{2}+\xi_{2}^{2}\right)+\frac{1}{2}\left(r^{2}+R^{2}\right),
$$

where $\delta= \pm 1$, which gives rise to the linear system of equations $\dot{z}=A_{0} z$, where

$$
z=\left(\begin{array}{l}
\xi_{1} \\
\xi_{2} \\
r \\
\eta_{1} \\
\eta_{2} \\
R
\end{array}\right), \quad A_{0}=\left(\begin{array}{cccccc}
0 & \tilde{\omega} & 0 & 0 & 0 & 0 \\
-\tilde{\omega} & 0 & 0 & 0 & 0 & 0 \\
0 & 0 & 0 & 0 & 0 & 1 \\
-\delta & 0 & 0 & 0 & \tilde{\omega} & 0 \\
0 & -\delta & 0 & -\tilde{\omega} & 0 & 0 \\
0 & 0 & -1 & 0 & 0 & 0
\end{array}\right) .
$$

Consider a smooth quadratic perturbation of $Q_{0}$, i.e., a quadratic Hamiltonian of the form $Q(\nu)=Q_{0}+\nu Q_{1}+\cdots$, where $\nu$ is the perturbation parameter. By Sokol'skii 33] (also see the discussion in [20], Chapter IV) there are four quantities that are important in the theory of normal forms for this problem, namely

$$
\Gamma_{1}=\xi_{2} \eta_{1}-\xi_{1} \eta_{2}, \quad \Gamma_{2}=\left(\xi_{1}^{2}+\xi_{2}^{2}\right) / 2, \quad \Gamma_{3}=\left(\eta_{1}^{2}+\eta_{2}^{2}\right) / 2, \quad I=\frac{1}{2}\left(r^{2}+R^{2}\right) .
$$

The higher order terms in $Q(\nu)$ are in normal form if they are functions of $\Gamma_{1}, \Gamma_{3}$ and $I$ only. Assume that $Q(\nu)$ is normalized through terms in $\nu$, so that $Q_{1}=$ $a \Gamma_{1}+b \Gamma_{3}+c I$ or

$$
Q(\nu)=\tilde{\omega} \Gamma_{1}+\delta \Gamma_{2}+I+\nu\left(\tilde{a} \Gamma_{1}+\tilde{b} \Gamma_{3}+\tilde{c} I\right)+\cdots .
$$

In the case under consideration here $\tilde{c}=0$, since $\pm i$ is always an exponent of a relative equilibrium. The characteristic polynomial of the linear system defined by $Q(\nu)$ is

$$
\left(\lambda^{2}+1\right)\left(\left\{\lambda^{2}+(\omega+\nu \tilde{a})^{2}\right\}^{2}+2 \nu \tilde{b} \delta\left\{\lambda^{2}-(\omega+\nu \tilde{a})^{2}\right\}+\nu^{2} \tilde{b}^{2} \delta^{2}+\cdots\right),
$$

which has roots

$$
\lambda= \pm i, \quad \lambda= \pm(\tilde{\omega}+\nu \tilde{a}) i \pm \sqrt{-\tilde{b} \delta \nu}+\cdots .
$$

So the coefficient " $\tilde{a}$ " controls the way the eigenvalues move in the imaginary direction, and the coefficient " $\tilde{b}$ " controls the way the eigenvalues split off the imaginary axis. The assumption that $\tilde{b} \neq 0$ means that the eigenvalues move off the imaginary axis when $\tilde{b} \delta \nu<0$.

Now consider a nonlinear Hamiltonian system depending on the parameter $\nu$ which has $Q(\nu)$ as its quadratic part and when $\nu=0$ has been put in Sokol'skii's normal form through the fourth order terms (see the discussion in Chapter VII of [20]), i.e., consider

$$
\begin{aligned}
\tilde{H}(\nu)=\tilde{\omega} & \Gamma_{1}+\delta \Gamma_{2}+I+\nu\left(\tilde{a} \Gamma_{1}+\tilde{b} \Gamma_{3}\right) \\
& +\frac{1}{2}\left(\tilde{d}_{1} \Gamma_{1}^{2}+\tilde{d}_{2} \Gamma_{3}^{2}+\tilde{d}_{3} I^{2}+\tilde{d}_{4} \Gamma_{1} \Gamma_{3}+\tilde{d}_{5} \Gamma_{2} I+\tilde{d}_{6} \Gamma_{3} I\right)+\cdots,
\end{aligned}
$$

where here the ellipsis stands for terms which are at least second order in $\nu$ or fifth order in the rectangular variables. 
The slight generalization of the main bifurcation in 22 is as follows - also see [20, 10].

Theorem. Consider a Hamiltonian of the form (15) with $\tilde{\omega}$ not equal to a small rational number, $\delta= \pm 1, \tilde{b} \neq 0, \tilde{d}_{2} \neq 0$. Then there is always a Lyapunov family of periodic solutions emanating from the origin with period close to $2 \pi$.

Case $A: \delta \tilde{d}_{2}>0$. The two additional Lyapunov families emanate from the origin when $\delta \tilde{b} \nu$ is small and positive. These families persist when $\nu=0$ as two distinct families of periodic orbits emanating from the origin. As $\delta \tilde{b} \nu$ becomes negative, the two families detach from the origin as a single family and recede from the origin.

Case $B: \delta \tilde{d}_{2}<0$. The two additional Lyapunov families emanate from the origin when $\delta \tilde{b} \nu$ is small and positive, and the families are globally connected. This global family shrinks to the origin as $\delta \tilde{b} \nu$ tends to zero through positive values. When $\delta \tilde{b} \nu$ is small and negative, there are no periodic solutions close to the origin.

One can compute the multipliers approximately to show that in Case A the periodic solutions are elliptic. In Case B, the periodic solutions are initially elliptic as they emanate from the origin, but go through extremal bifurcations to become hyperbolic-elliptic.

To apply this theorem to the reduced problem let $\nu=\mu-\tilde{\mu}_{1}(\varepsilon)$, so for fixed small $\varepsilon$ the Lagrange relative equilibrium of the reduced problem has exponents $\pm i$ with multiplicity one and $\pm i \tilde{\omega}(\varepsilon)$ with multiplicity two, where $\tilde{\omega}=\sqrt{2} / 2+$ $O(\varepsilon)$. So for fixed small $\varepsilon$ the Hamiltonian of the reduced problem at the Lagrange relative equilibrium can be put into Sokol'skii's normal form (15). When $\varepsilon=0$ the important coefficients have been calculated in the restricted problem, namely $\tilde{a}(0)=-3 \sqrt{2} \sqrt{69} / 16, \tilde{b}(0)=-3 \sqrt{69} / 8$ (see [29]), and $\tilde{d}_{2}(0)=59 / 108$ (see [30]). So for small $\varepsilon, \tilde{b}(\varepsilon)$ and $\tilde{d}_{2}(\varepsilon)$ are non-zero and have the same sign. Thus:

Theorem. For small $\varepsilon$ the reduced problem undergoes the bifurcation given in Case $A$ of the above theorem at the Lagrange relative equilibrium as $\mu$ passes through $\tilde{\mu}_{1}(\varepsilon)$.

5.4. Bridges and natural centers. There are many different bifurcations from the two Lyapunov families of periodic solutions which emanate from $\mathcal{L}_{4}$ as the parameter $\mu$ is varied. In particular, very interesting bifurcations occur as the parameter $\mu$ passes through the values $\mu_{p / q}$, where $p / q$ is a rational number.

The careful numerical work of Deprit, Henrard and Palmore [9, 25] found families of periodic solutions, called bridges, connecting the two Lyapunov families at $\mathcal{L}_{4}$. In general, a bridge consisted of two families of periodic solutions, of which one is elliptic and the other hyperbolic. As the parameter $\mu$ is varied, a particular bridge would collapse into the equilibrium $\mathcal{L}_{4}$ as $\mu \longrightarrow \mu_{p / q}$.

Using the computations of the normal form at $\mathcal{L}_{4}$ by Deprit and Deprit-Bartholomé [8], Meyer and Palmore [21] were able to establish the existence of these bridges using a variation of Birkhoff's fixed point theorem. As with most fixed point arguments, there were no uniqueness or continuity conclusions. Finally, Schmidt [28] proved a series of theorems for general Hamiltonian systems which explain almost all the local bifurcations observed in the restricted problem in 92 25]. These theorems have been reproved many times; see [10] for additional references.

Using the same line of argument, one can show that for small $\varepsilon$ the reduced problem has bridges and natural centers which are continuations of the bridges and natural centers found in the restricted problem. There are surely many more 
bifurcations in the spatial restricted problem, due to the third Lyapunov family in the third direction, but that analysis remains to be done.

\section{Continuation of KAM tori}

There are several examples where the classical KAM theorems have been applied to the restricted problem, but we know of only one case where our method applies at present. That one case is in the planar problem, so we will only consider the planar problem now. Because the planar restricted problem has two degrees of freedom, one obtains not only the existence of invariant tori, but some stability information also. For the three degree of freedom reduced problem the existence of the invariant tori will not imply any stability information.

We will say that a nondegenerate $\tau$-periodic solution $p(t)$ of the restricted problem with $H_{R}(p(t))=h_{0}$ is of general elliptic type if the cross section map for this periodic orbit is of the form

$$
\begin{aligned}
& \Psi^{\prime}=\Psi+\cdots, \\
& \psi^{\prime}=\psi+\omega+\alpha h+\beta \Psi+\cdots, \\
& T=\tau+\gamma h+\delta \Psi+\cdots,
\end{aligned}
$$

where the constants $\alpha, \beta, \gamma, \delta$ satisfy

$$
\alpha \delta-\beta \gamma \neq 0,
$$

$\tau$ is not a multiple of $2 \pi$, and $\omega$ is irrational. In the above $h=H_{R}-h_{0}, \Psi, \psi$ are action-angle coordinates in the cross section in the level set $H_{R}=h_{0}+h$, and $T$ is the first return time for the cross section. The periodic solution, $p$, corresponds to the point $\Psi=0$ when $h=0$.

Since the periodic solution is nondegenerate $(\tau \omega \neq 2 \pi k, k \in \mathbb{Z})$ and $\tau$ is not a multiple of $2 \pi$, the periodic solution can be continued into the reduced problem for small $\varepsilon$ by the results of Section 3 As we shall see, additional assumptions are needed to apply one of the standard KAM theorems to the continuation of this periodic solution.

Theorem. Let $\tilde{p}(t, h, \varepsilon)$ be the continuation into the reduced problem of the general elliptic periodic solution $p(t)$ of the restricted problem. For $\varepsilon$ small and $h$ near $h_{0}$ the periodic solution $\tilde{p}(t, h, \varepsilon)$ is the limit of invariant three-dimensional tori (KAM tori).

Proof. Since the Hamiltonian of the reduced problem is $H=H_{R}+I+O(\varepsilon)$, we can compute the cross section map for the periodic solution $\tilde{p}(t, h, 0)$ (the continuation periodic solution for $\varepsilon=0)$. Use $\Psi, I, \psi, \iota$ as coordinates in this cross section. Setting $H=h_{0}$ is the same as setting $H_{R}=h_{0}+h$ and $I=-h$. The section map becomes

$$
\begin{array}{ll}
\Psi^{\prime}=\Psi+\cdots, & \psi^{\prime}=\psi+\omega-\alpha I+\beta \Psi+\cdots, \\
I^{\prime}=I+\cdots, & \iota^{\prime}=\iota+\tau-\gamma I+\delta \Psi+\cdots .
\end{array}
$$

The assumption $\alpha \delta-\beta \gamma \neq 0$ assures that the standard KAM theorems apply. So the section map admits invariant two-dimensional tori which limits onto the fixed point which means that the full problem admits invariant three-dimensional tori. Of course this no longer implies that the periodic solution is orbitally stable. 
Using the normal form calculation of Deprit and Deprit-Bartholomé 8, Meyer and Palmore [21] computed the cross section map to the Lyapunov periodic solutions and showed that there was a "twist". Let $\mu_{n}$ denote the mass ratio at which $\omega_{1} / \omega_{2}=n$ and $\mu_{n} \leq 1 / 2$, where $n$ is a positive integer. The explicit formula for $\mu_{n}$ is given in (14), but for now note that $\mu_{n+1}<\mu_{n}$. Provided $0<\mu<\mu_{1}$, $\mu \neq \mu_{2}, \mu_{3}, \mu_{4}$, the Hamiltonian of the restricted problem at $\mathcal{L}_{4}$ can be brought into the normal form

$$
H\left(J_{1}, J_{2}, \psi_{1}, \psi_{2}\right)=\omega_{1} J_{1}-\omega_{2} J_{2}+\frac{1}{2}\left\{A J_{1}^{2}+2 B J_{1} J_{2}+C J_{2}^{2}\right\}+\cdots,
$$

where $J_{1}, J_{2}, \psi_{1}, \psi_{2}$ are action-angle variables and

$$
\begin{gathered}
0<\omega_{2}<\frac{\sqrt{2}}{2}<\omega_{1}, \\
\omega_{1}^{2}+\omega_{2}^{2}=1, \\
\omega_{1}^{2} \omega_{2}^{2}=27 \mu(1-\mu) / 4 .
\end{gathered}
$$

When $\mu=\mu_{2}, \mu_{3}, \mu_{4}$ there are other terms in the normal form that appear at the same level of truncation - see [20].

In the classic paper by Deprit and Deprit-Bartholomé $[8]$ the constants of the normal form were found to be

$$
\begin{gathered}
A=\frac{\omega_{2}^{2}\left(81-696 \omega_{1}^{2}+124 \omega_{1}^{4}\right)}{72\left(1-2 \omega_{1}^{2}\right)^{2}\left(1-5 \omega_{1}^{2}\right)}, \\
B=-\frac{\omega_{1} \omega_{2}\left(43+64 \omega_{1}^{2} \omega_{2}^{2}\right)}{6\left(4 \omega_{1}^{2} \omega_{2}^{2}-1\right)\left(25 \omega_{1}^{2} \omega_{2}^{2}-4\right)}, \\
C\left(\omega_{1}, \omega_{2}\right)=A\left(\omega_{2}, \omega_{1}\right) .
\end{gathered}
$$

Theorem. If $0<\mu<\mu_{1}, \mu \neq \mu_{2}, \mu_{3}, \mu_{4}$, then the continuations into the reduced problem of the short and long period families of the restricted problem are the limit of invariant three-dimensional tori (KAM tori).

Proof. The equations are

$$
\begin{aligned}
& \dot{J}_{1}=0+\cdots, \quad \dot{J}_{2}=0+\cdots, \\
& \dot{\psi_{1}}=-\omega_{1}-\left\{A J_{1}+B J_{2}\right\}+\cdots, \\
& \dot{\psi_{2}}=\omega_{2}-\left\{B J_{1}+C J_{2}\right\}+\cdots .
\end{aligned}
$$

$\psi_{2}=0$ is a cross section to the flow, and the first return time is

$$
T=\frac{2 \pi}{\omega_{2}-\left\{B J_{1}+C J_{2}\right\}}=\frac{2 \pi}{\omega_{2}}\left\{1+\frac{1}{\omega_{2}}\left\{B J_{1}+C J_{2}\right\}+\cdots\right\} .
$$

Use $J=J_{1}$ and $\psi=\psi_{1}$ as coordinates in the cross section $\psi_{2}=0$ in the energy level $H=h$. Let the section map be $(J, \psi) \rightarrow\left(J^{\prime}, \psi^{\prime}\right)$. Compute

$$
\begin{aligned}
\psi^{\prime} & =\psi+\left\{-\omega_{1}-\left(A J+B J_{2}\right)\right\} T \\
& =\psi-2 \pi \frac{\omega_{1}}{\omega_{2}}-\frac{2 \pi}{\omega_{2}^{2}}\left\{\left(B \omega_{1}+A \omega_{2}\right) J+\left(B \omega_{2}+C \omega_{1}\right) J_{2}\right\}+\cdots .
\end{aligned}
$$


Now use $H=h$ to eliminate $J_{2}$ by substituting

$$
J_{2}=\frac{\omega_{1}}{\omega_{2}} J-\frac{h}{\omega_{2}}+\cdots,
$$

to get

$$
\begin{aligned}
& J^{\prime}=J+\cdots, \\
& \psi^{\prime}=\psi-\frac{\omega_{1}}{\omega_{2}} 2 \pi-\frac{2 \pi}{\omega_{2}^{3}}\left\{\left(A \omega_{2}^{2}+2 B \omega_{1} \omega_{2}+C \omega_{1}^{2}\right) J-\left(C \omega_{1}+B \omega_{2}\right) h\right\}+\cdots, \\
& T=\frac{2 \pi}{\omega_{2}}\left\{1+\frac{1}{\omega_{2}^{2}}\left\{\left(B \omega_{2}+C \omega_{1}\right) J-C h\right\}\right\}+\cdots
\end{aligned}
$$

This section map is of the form discussed above, and

$$
\begin{aligned}
\alpha \delta-\beta \gamma & =-\left(\frac{2 \pi}{\omega_{2}^{2}}\right)^{2}\left(A C-B^{2}\right) \\
& =-\left(\frac{2 \pi}{\omega_{2}^{2}}\right)^{2} \frac{\sigma\left(-107172-3298947 \sigma+8799272 \sigma^{2}+384400 \sigma^{3}\right)}{5184(4 \sigma-1)^{2}(25 \sigma-4)^{2}}
\end{aligned}
$$

with $\sigma=\omega_{1}^{2} \omega_{2}^{2}$.

The numerator has obviously only one positive real root, which is found by numerical methods to be $\sigma \approx 0.398543$. The value of $\sigma$ at $\mu_{1}$ is $\sigma=.25$, so that the numerator is nonzero for $0<\mu<\mu_{1}$. (The referee pointed out that we did not need numerics. Let $N(\sigma)$ be the nontrivial factor of the numerator. Then $N(0)<0$, $N^{\prime}(0)<0, N^{\prime \prime}>0, N(1 / 4)<0$ prove that $N$ has no zeros in $(0,1 / 4)$. Thanks.) The denominator is zero for $\mu_{1}$ and $\mu_{2}$, but these values are excluded since the normalization does not hold. Thus $\alpha \delta-\beta \gamma$ is defined and nonzero for $0<\mu<\mu_{1}$, $\mu \neq \mu_{2}, \mu_{3}, \mu_{4}$, and the theorem follows.

\section{REFERENCES}

[1] R. Abraham and J. Marsden, Foundations of Mechanics, Benjamin Cummings, London, 1978. MR 81e:58025

[2] V. I. Arnold (Ed.), Encyclopaedia of Mathematical Sciences, Dynamical Systems III, Springer-Verlag, 1993. MR 95d:58043a

[3] E. A. Belbruno, A new family of periodic orbits for the restricted problem, Celestial Mech., 25, 1981, 397-415. MR 82m:70017

[4] G. D. Birkhoff, The restricted problem of three-bodies, Rend. Circolo Mat. Palermo, 39, 1915, 255-334.

[5] — Dynamical Systems, Amer. Math. Soc., Providence, RI, 1927.

[6] D. Buchanan, Trojan satellites - limiting case, Trans. Roy. Soc. Canada Sect. III (3), 35, 1941, 9-25. MR 3:216a

[7] C. C. Conley, Some new long period solutions of the plane restricted body problem of threebodies, Comm. Pure Appl. Math., 16, 1963, 449-467. MR 27:4670

[8] A. Deprit and A. Deprit-Bartholomé, Stability of the Lagrange points, Astron. J., 72, 1967, 173-179.

[9] A. Deprit and J. Henrard, A manifold of periodic solutions, Advan. Astron. Astrophy, 6, $1968,6-12$.

[10] M. Golubitsky, J. Marsden, I Stewart, and Michael Dellnitz, The constrained LyapunovSchmidt procedure and periodic orbits, Fields Institute Communications, Vol. 4, 81-127, 1995. MR 96g:58135

[11] J. D. Hadjidemetriou, The continuation of periodic orbits from the restricted to the general three-body problem, Celestial Mech., 12, 1975, 155-174. MR 52:7243 
[12] A. Liapounoff [Lyapunov], Problème générale de la stabilité du mouvement, Ann. Math. Studies 17, Princeton Univ. Press, Princeton, NJ, 1947. (Reproduction of the French translation.) MR 9:34j

[13] J. Marsden and A. Weinstein, Reduction of symplectic manifolds with symmetries, Rep. Math. Phy., 5, 1974, 121-130. MR 53:6633

[14] K. R. Meyer, Comet like periodic orbits in the N-body problem, J. Comp. and Appl. Math., 52, 1994, 337-351. MR 95k:70026

[15] _ Generic bifurcation of periodic points, Trans. Amer. Math. Soc., 149, 1970, 95-107. MR 41:3931

[16] - Generic stability properties of periodic points, Trans. Amer. Math. Soc., 154, 1971, 273-277. MR 42:6373

[17] _ Periodic orbits near infinity in the restricted $N$-body problem, Celest. Mech., 23, 1981, 69-81. MR 82c:70015

[18] Periodic solutions of the N-body problem, J. Dif. Eqs., 39(1), 1981, 2-38. MR 83b:70016

[19] Symmetries and integrals in mechanics, Dynamical Systems (M. Peixoto, Ed.), Academic Press, New York, 1973, 259-272. MR 48:9760

[20] K. R. Meyer and G. R. Hall, Introduction to Hamiltonian Dynamical Systems and the N-body Problem, Springer-Verlag, New York, 1992. MR 93b:70002

[21] K. R. Meyer and J. I. Palmore, A new class of periodic solutions in the restricted three-body problem, J. Diff. Eqs., 44, 1970, 263-272. MR 46:7640

[22] K. R. Meyer and D. S. Schmidt, Periodic orbits near $\mathcal{L}_{4}$ for mass ratios near the critical mass ratio of Routh, Celest. Mech., 4, 1971, 99-109. MR 47:6164

[23] F. R. Moulton, A class of periodic solutions of the problem of three-bodies with applications to lunar theory, Trans. Amer. Math. Soc., 7, 1906, 537-577.

[24] A class of periodic orbits of the superior planets, Trans. Amer. Math. Soc., 13, 1912, 96-108.

[25] J. I. Palmore, Bridges and Natural Centers in the Restricted Three-Body Problem, University of Minnesota Report, Minneapolis MN, 1969. MR 43:8279

[26] O. Perron, Neue periodische Lösungen des ebenen Drei und Mehrkörperproblem, Math. Z., 42, 1937, 593-624.

[27] H. Poincaré, Les méthodes nouvelles de la mécanique céleste, Gauther-Villars, Paris, 1899. MR 89e:01054 (reprint)

[28] D. S. Schmidt, Periodic solutions near a resonant equilibrium of a Hamiltonian system, Celest. Mech., 9, 1974, 81-103. MR 49:9340

[29] Transformation to versal normal form, Computer Aided Proofs in Analysis, (Ed. K. R. Meyer and D. S. Schmidt), IMA Series 28, Springer-Verlag, 1990, 235-240. MR 92b:58210

[30] Wersal normal form of the Hamiltonian function of the restricted problem of threebodies near $\mathcal{L}_{4}$, J. Computational and Appl. Math., 52, 1994, 155-176. MR 95k:70020

[31] C. L. Siegel, Über eine periodische Lösung im Dreikörperproblem, Math. Nachr. , 4, 1950, 28-64. MR 14:910d

[32] C. L. Siegel and J. K. Moser, Lectures on Celestial Mechanics, Springer-Verlag, New York, 1971. MR 58:19464

[33] A. Sokol'skii, On the stability of an autonomous Hamiltonian system, J. Appl. Math. Mech., 38, 1978, 741-49. MR 57:10734

[34] E. T. Whittaker, A Treatise on the Analytical Dynamics of Particles and Rigid Bodies, Cambridge University Press, 1927.

Department of Mathematical Sciences, University of Cincinnati, Cincinnati, Ohio 45221-0025

E-mail address: ken.meyer@uc.edu

Department of Electrical and Computer Engineering and Computer Science, UniVersity of Cincinnati, Cincinnati, Ohio 45221-0030

E-mail address: dieter.schmidt@uc.edu 